\title{
VIII.
}

\section{Ein seltener Fall von beinahe universellem angebornem fortschreitendem Riesenwuchs.}

(Aus der Nervenklinik des Communehospitals in Kopenhagen. Oberarzt, Professor Dr. Kn ud Pontoppidan.)

\author{
Von Dr. D. E. Jacobson, \\ Privatdocenten, I. Assistenzarzt.
}

Ein 3jähriges Mädchen wurde am 13. Juli 1892 in die Nervenklinik unter der recht ungewöhnlichen Diagnose, Akromegalie, aufgenommen. Es zeigte sich schnell, dass die Akromegalie, wovon hier die Rede war, nichts mit derjenigen Krankheit zu thun hatte, welche Marie $\left.{ }^{1}\right) 1886$ entdeckte und unter dieser Bezeichnung veröffentlichte. Diese Krankheit besteht, wie bekannt, ganz gewiss in einer abnorm starken Entwickelung gewisser Körpertheile, besonders peripherischer (Nase, Unterlippe, Unterkiefer, Ohren, Hände, Füsse, u. s. w.), allein - und das ist Punctum saliens - dieser Riesenwuchs entsteht bei früher völlig normalen Individuen etwa im Pubertätsalter oder später. Es wurde dagegen in unserem Falle bald klar, dass es sich um eine eigentliche Krankheit gar nicht handelte, sondern um eine angeborne Missbildung, eine Entwickelungsanomalie, einen Riesenwuchs, der den grössten Theil des Körpers der kleinen Patientin umfasste. Indem ich mich nachher mit der dahin gehörenden Literatur beschäftigte, wurde ich schnell davon überzeugt, dass das Schicksal uns eine sehr grosse pathologische Seltenheit - um nicht geradeza ein Unicum zu sagen - in die Hände gespielt hatte.

Deshalb habe ich einen sehr eingehenden Krankenbericht aufgenommen, den ich mit der freundlichen Erlaubniss des Herrn Professor Pontoppidan veröffentliche, um dadurch das weitest mögliche Interesse für diesen merkwürdigen Fall zu erwecken, welcher hier im Hospitale allgemeines Aufsehen erregt hat.

1) Revue de médecine, VI. 1886. 
Um den Text näher zu erklären, füge ich den Holzschnitt nach einem photographischen Bilde bei, das trotz der Unruhe und des Weinens des Kindes ganz gut ausgefallen war.

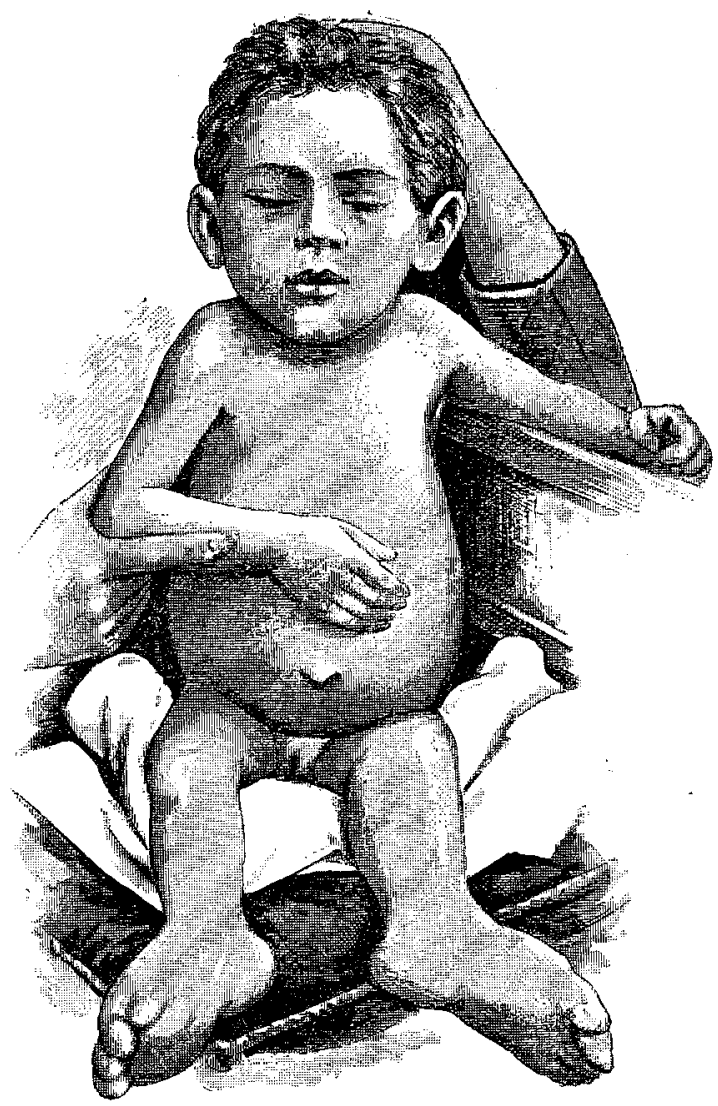

Carla H., 3 Jahre alt, Tochter eines unverheiratheten Ladenfräuleins. Aufgenommen 13. Juli 1892.

Keine hereditäre Disposition. Keine Missbildung in den Familien der Eltern. Weder der Vater noch die Mutter (ich habe mit beiden gesprochen) haben Syphilis gehabt, noch sind sie Alkobolisten. Sie sind alle beide jung, von Mittelgrösse und ungefähr 22 Jahre alt. Der Zustand der Mutter während der Gravidität ist natürlicb gewesen; die Geburt verlief normal.

Schon bei der Geburt ist das Aeussere des Kindes so auffällig gewesen, dass es der Mutter nicht erlaubt wurde, es gleich zu sehen. Da die unverheiratbete Mutter in einer der Filialen unserer öffentlichen Gebäranstalt ent- 


\section{6}

bunden wurde, so baben wir im Protocolle der Anstalt eine Quelle, woraus ganz sichere Auskünfte zu schöpfen sind. Am Geburtstage des Kindes ist Folgendes zn Protocoll genommen: "In der Filiale N. N. wurde heute ein ausgetragenes lebendiges Mädchen geboren; es $w 0 g 3250 \mathrm{~g}$, maass $51 \mathrm{~cm}$, hatte ausgebreitete Teleangiektasion an der Brust und an Unterkörper, sammt Makropodie".

Nach der Geburt ist das Kind in die Pflege gegeben und dort wahrscheinlich etwas vernachlässigt worden. Bei der Aufnahme in's Hospital war es etwas rachitisch, batte Fieber und stinkende grünlicbe Oeffnung. Nach einigen Tagen schwand indessen das Fieber; der Stuhlgang kam in Ordnung, und nachher ist das Kind gut gediehen und hat stets an Gewicht zugenommen.

Nach Verlauf ron ungefähr einer Woche war der Status praesens, wie folgt:

Sogar ganz oberflächlich betrachtet, weicht das Aeussere des Kindes in merkwürdiger Weise von der Norm ab. Die rechte Wange, der rechte Arm und die rechte Hand, die linke Hälfte der Brust und des Unterleibes, das rechte Labium majus, das linke Bein, sowie in ganz besonderem Grade die beiden Füsse zeigen nehmlich eine augenfällige Vergrösserung. Dieser „Riesenwucbs" erscheint um so stärker, als das Kind sonst ziemlich klein im Verhältniss zu seinem Alter, zart gebaut, mager, schlaff und kränklich ist (Rachitis). Die Hautfarbe ist endlich auch eine Erscheinung, welche gleich die Aufmerksamkeit erregt. An dem Truncus, - sowohl an der Vorderals an der Hinterfläche -, und den Extremitäten hat die Haut in einem weiten Umfange eine röthlich-violette Farbe. Dieselbe erscheint am stärksten, wenn das Kind schreit; es erhält dann ein förmlich marmorirtes Aussehen, indem die gefärbten Theile der Haut gegen die normal blassen contrastiren. Die röthlich-violette Farbe rührt von capillären Teleangiektasien in der Haut her.

Eine mehr detaillirte Untersuchung erweist Folgendes:

Gewicht $8450 \mathrm{~g}$.

Länge vom Scheitel bis zum rechten Fersenrand $78 \mathrm{~cm}$

- linken - 80 -

Der Ernährungszustand schlecht; die Musculatur äberall schlaff, die subcutane Fettlage äusserst gering (ausgenommen an den Füssen). Das Kind ist von Gemüth verblüffend ernsthaft; man sieht es nie lächeln. Es versucht auch nie zu sprechen, obgleich es recht intelligent scheint. Es versteht z. B. Alles, was man ihm sagt, und scheint grosses Interesse an den verschiedenen Untersuchungsmethoden zu haben, ja ist sogar damit behülflich, indem es oft Finger, Füsse u. dgl. zum Messen hervorstreckt. Es mag gern spielen und scheint grossen Werth darauf zu legen, wenn man es liebkost, wie auch auf die mehr materiellen Güter des Lebens; sein Lieblingsgetränk ist Wein und Wasser, wozu es beinahe die Neigung des Alkoholisten empfindet. Obgleich 3 Jahre alt, kann es nicht reinlich gehalten werden. 
Der Kopf scheint nicht grösser, als gewöhnlich bei rachitischen Kindern, die rechte Hälfte ist aber merklich umfangreicher, als die linke, namentlich steht die rechte Pars occipitalis als ein grosser Buckel hervor.

Der Umfang des Hirnschädels in der Höhe der Arcus superciliares und der Protuberantia occipitalis beträgt $47 \mathrm{~cm}$;

$$
\begin{aligned}
& \text { die rechte Bälfte misst. . . . . } 24 \frac{1}{2} \mathrm{~cm} \\
& \text { - linke - - . . . . } 22 \frac{1}{2} \text { - } \\
& \text { der grösste Längendurchmesser . . 15,8 - } \\
& \text { - - Breitendurchmesser . . 13,4 - }
\end{aligned}
$$

Das Gesicht ist rundlich; die rechte Wangengegend springt aber über das übrige Niveau stark hervor, so dass die Patientin aussieht, als hätte sie ein grosses Zahngeschwür. Das Verzerrte in ihrem Aussehen wird noch merkbarer dadurch, dass der rechte Mundwinkel stark herabbängt. Der Abstand vom Filtrum bis zum rechten Ohrzipfel beträgt $10,5 \mathrm{~cm}$, bis zum linken nur $9,8 \mathrm{~cm}$. Die Verdickung der Wange ist in überwiegendem Grade von den subcutanen Weichtheilen bedingt, ohne dass es sich näher bestimmen lässt, welchen Antheil die Muskeln daran haben. Es ist indessen auch eine kleine Vergrösserung des rechten Theiles des Oberkiefers vorhanden, und das rechte 0 s zygomaticum, wie auch der rechte Ramus horizontalis des Unterkiefers, fühlen sicb etwas massiver an, als die gleichnamigen Knochen der linken Seite. Auch ist die rechte Hälfte der Oberlippe ein wenig voller, als die linke.

Die grösste Dicke der Wange ist

$$
\begin{array}{lll}
\text { an der rechten Seite. } & . & 1,6 \mathrm{~cm} \\
\text { - }- \text { linken - . } & 0,9
\end{array}
$$

Der Abstand von der Spina mentalis bis zu den Anguli maxillae ist gleich gross an beiden Seiten $=7 \mathrm{~cm}$.

Der übrige Theil des Gesichts ist woblgebildet; die Augenlider sind nicht bauschig, die Augenbranen gut entwickelt, die Nase klein und fein gebildet, die Unterlippe nicht dick und herabhängend; die Angen sind natürlich, wie auch die Ophthalmoskopie zeigt; die Ohren sind etwas gross, aber gut geformt und von gleicher Länge, nehmlich $5 \mathrm{~cm}$; das Gehör scheint gut. Die Zähne sind in passender Anzabl da, aber stark cariös. Die Zunge bietet nichts Abnormes dar, eben so wenig die Mundschleimhaut, der Schlund und der Gaumen, jedoch ausgenommen, dass der rechte vordere Gaumenbogen ein wenig voller ist, als der linke. Keine abnorme Salivation. Der Haarwuchs des Hauptes reichlich. Der Hals ist von natürlicher Länge und Dicke. Keine Vergrösserung der Schilddrüse; dagegen grössere, indolente Drüsengeschwülste an beiden Regiones laterales colli. Keine Dämpfung über der Thymus. Die rechte Oberextremität ist in toto grösser.und voller, als die linke; dieses betrifft jedoch den Vorderarm mehr, als den Oberarm. Am meisten betrifft die Vergrösserung jedoch die Hand, wo der Zeigefinger im Vergleich mit den anderen Fingern besonders begünstigt worden ist. Die Vergrösserung der Extrenitäten rührt von einer gleichmässigen Vergrösserung der Weichtheile und der Skelettheile her; nur die Haut ist, was 
die Consistenz betrifft, weder verdickt, noch verändert. Der Umfang der Epiphysen scheint nicht stärker vergrössert zu sein, als der Umfang der Diaphysen, auch ist keine auffällige Hyperplasie des subcutanen Gewebes vorhanden. Die Nägel sind bis auf ihr ein wenig grösseres Volumen ganz natürlich. Die Harmonie der verschiedenen Theile des Arms ist, mit Ausnahme des im Verhältniss zu den anderen Fingern zu grossen Zeigefingers, bewahrt.

Die Grössenverhältnisse werden übrigens aus nachstehenden Maassangaben näher hervorgehen:

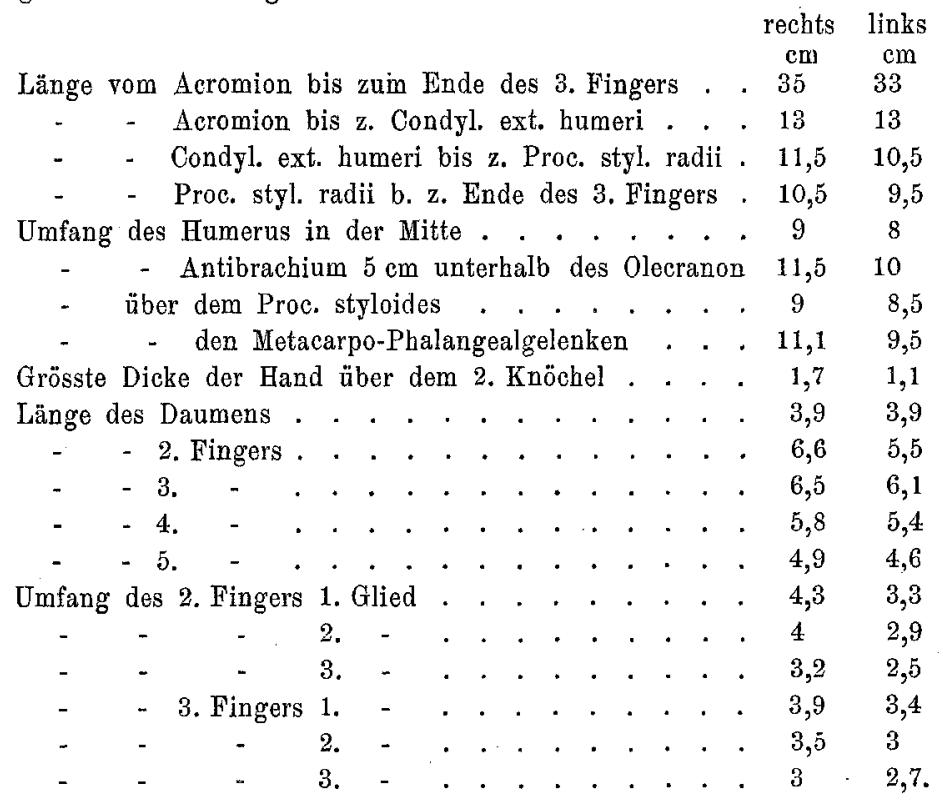

Das Kind kann die Arme frei gebrauchen und benutzt gern den rechten mehr, als den linken. Scapulae und Claviculae sammt Sternum nicht verdickt, nur rachitischer Rosenkranz. Die linke Brusthälfte ist indessen ein wenig voluminöser, als die recbte.

Umfang in der Höhe der rechten Brustwarze $21 \mathrm{~cm}$ - linken - 23 -

Die Brustorgane bieten nichts Besonderes dar. Der Unterleib ist sehr gross, mit gespannter Wand und dünner praller Haut. Weder die Leber noch die Milz lassen sich unter der Curvatur herauspercutiren oder palpiren.

Die linke Hälfte des Unterleibes ist etwas voluminöser, als die rechte.

Umfang in der Höhe rom Umbilicus, rechte Hälfte $26,25 \mathrm{~cm}$ linke - 30,75 -

Die Beckenknochen sind nicht verdickt. Das rechte Labium majus etwas grösser und stärker, als das linke.

Columna vertebr. nicht skoliotisch; allein in der Lumbo-Dorsalgegend bil- 
det sie eine abgerundete, nieht stark prominirende Kypbose (rachitisch?), deren Scheitelpunkt vom Proc. spin. vert. dors. XII gebildet wird. Die Kyphose lässt sich nicht ganz gerade machen. Die linke Unterextremität ist in toto länger und stärker, als die rechte. Merkwürdig genug bat das Femur den grössten Antheil an der Verlängerung. Sowohl die knöchernen Theile, als die Weichtheile bedingen den vergrösserten Dmfang, besonders aber die ersteren. Das ganze linke Knie (mit Patella) ist voluminöser, als das recbte; namentlich ist der Condylus internus stark entwickelt, so dass eine bedeutende Valgusstellung vorbanden ist, die doch auch, wenn gleich weniger ausgesprochen, an der rechten Seite vorkommt. Es findet keine abnorme Krümmung der langen Knochen statt.

Die Länge der Unterextremität vom oberen Rande des Troch. major bis zur Spitze des Mall. ext. . . . . . . . 3031 Troch. maj. bis zur Kniegelenklinie . . . . . . . 1517 Kniegelenk bis zur Spitze des Mall. ext. . . . . . . $15 \quad 15,5$ Umfang des Femur in der Mitte. . . . . . . . . . 14 15,5

- $\quad$ - Knies quer über Patella . . . . . . . . 15,4 16,7

- - UnterschenkeIs, $5 \mathrm{~cm}$ unterbalb des Apex patellae $13 \quad 15$ über den Malleoli . . . . 11,5 12,5.

Die linke Patella ist breiter und auch länger, als die rechte.

$\begin{array}{cc}\text { rechts } & \text { links } \\ \mathrm{cm} & \mathrm{cm} \\ 2,7 & 2,9 \\ 2,3 & 3 .\end{array}$

Die Füsse sind höchst eigenthümlich entwickelt. Sie sind sowohl zu gross, als auch zu breit und zu dick im Verbältniss zur Grösse und zum Alter des Kindes. Deckte man das Kind der Art zu, dass nur die Füsse zu sehen wären, so müsste man nach deren Länge auf ein 8jäbriges Kind rathen. Die Verdickung rülrt theilweise von der Haut ber, theilweise und namentlich ron dem subcutanen Fettgewebe, welches an Dorsum und in Planta weiche dicke Kissen bildet. Es lässt sich wegen des reichen Fettpolsters nicht genau bestimmen, welchen Antheil die Knochen an dem vergrösserten Volumen haben. Der linke Fuss ist im Ganzen genommen ein wenig grösser, als der rechte. Die Zehen sind in verschiedenem Grade entwickelt; durehgehend aber steht ihre Grösse in passendem Einklang mit der Grösse des Fusses. Die grossen Zeben, namentlich die grosse Zehe am linken Fusse, sind so gross, dass sie gut hübschen kleinen Damenfüssen gehören könnten. Die $z$ weite Zehe ist in die Länge und Breite gleichfalls stark entwickelt. Z wischen der ersten und 2 weiten Zehe befindet sich ein ungewöhnlich breiter Raum ( $4 \mathrm{~mm}$ am rechten, $3 \mathrm{~mm}$ am linken Fusse), als ob hier eine Zehe fehlte. Die fünfte Zehe ist unverbältnissmässig klein, besonders die linke. Die Nägel sind mit ihren bezw. Gliedern proportional entwickelt. Im Bette werden beide Füsse mit der Planta gegen einander ge- 
balten, wie in starker Varusstellung. Das Kind ist im Stande, beide Beine in die Höhe zu beben und die Füsse, wie Zehen, zu bewegen; es steht auch gut auf den Beinen und die Füsse ruhen alsdann mit ganzer Planta auf dern Fussboden; gehen kann es aber nicht; macht man den Versuch, so wird sich stets nur das rechte Bein vorwärts bewegen, nie das grössere linke. Die Patellarreflexe sind natürlich; die Plantarreflexe dagegen sehr schwach. Keine Oedeme.

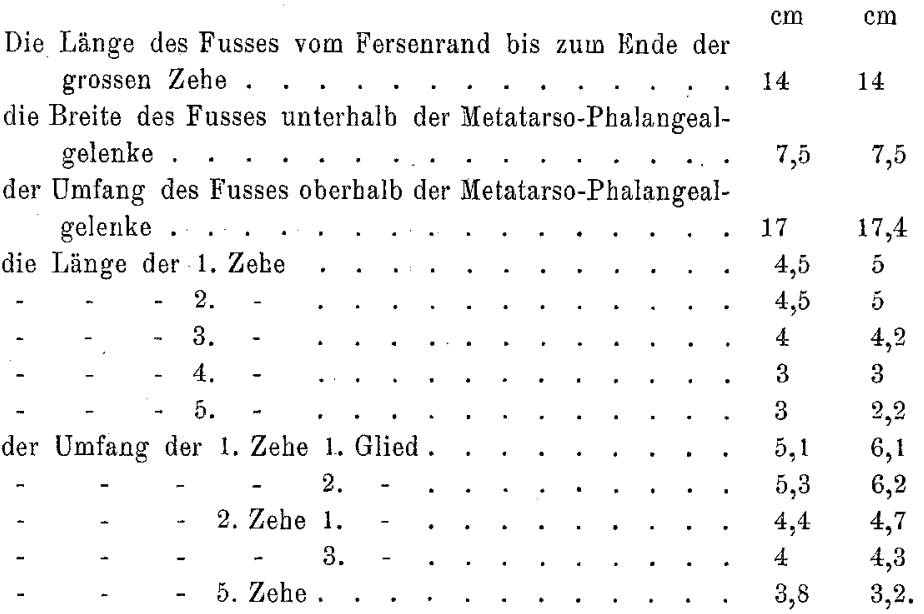

Faradische Reaction sämmtlicher Muskeln normal. Die Sensibilität scheint unbeschädigt.

Wie bereits berührt, finden sich in der Haut am Truncus (sowohl rechts als auch, und namentlich, links) und an den Extremitäten ausgedehnte, röthlich-violette, capilläre Teleangiektasien, welche sich scharf von den übrigen spärlichen und kleinen normalen Hautpartien abgrenzen, deren Farbe sie erbalten, wenn man mit dem Finger das Blut zur Seite drückt. Die normalen Hautinseln sind nicht symmetrisch. Es findet sich eine an der rechten Seite des Unterleibes; die Grenze nach innen zwischen dieser Insel und der gefärbten Partie an der linken Seite fällt genau in die Mitte.

Ausser diesen capillären Erweiterungen finden sich auch rund herum bervortretende oberflächliche Venennetze, besonders an der linken Seite des Unterleibs und der Brust und an dem linken Femur, wo die Venen hie und da varicös sind.

Am Nacken, in der Höhe der Vertebra prominens, und an der Hinterseite der Sebultergelenke finden sich kleine Inseln mit abnorm starkem Haarwuehs.

Nirgends abnorme Pigmentablagerung. Der Urin enthält weder Zucker noch Alburin.

Der Puls der grossen Arterien ist an beiden Seiten gleich.

Keine abnorme Schweisssecretion, ausgenommen am Kopfe (Rachitis!). 
Nach dem Lesen obiger Krankengeschichte wird mir wahrscheinlich Jeder darin Recht geben, dass wir hier auf einen sehr schön ausgesprochenen Fall abnorm vergrösserter WachsthumsEnergie gestossen sind. Es handelt sich nehmlich um eine schon bei der Geburt constatirte, abnorm starke Entwickelung, nicht nur einiger Finger und Zehen, ja eines ganzen Fusses, sondern um eine ähnliche Entwickelung gewisser Theile des Kopfes, des Gesichts, der Genitalien, der ganzen linken Seite des Truncus, endlich auch und namentlich um die Vergrösserung einer ganzen Ober- und Unterextremität, - welche Extremitäten nicht einmal gleichseitig sind, - und schliesslich noch um die Vergrösserung beider Füsse.

Ist dieses nun auch eine ausserordentliche Seltenheit?

Es gehört ja keineswegs zu den aussergewöbnlichen Vorkommnissen, den Riesenwuchs (Gigantophytie) einiger Finger oder Zehen (Makrodaktylie), ja eines ganzen Fusses (Makropodie), einer Hand oder noch grösserer Theile einer einzelnen Extremität zu treffen. Es liegen ein Paar recht umfangreiche Statistiken vor: Wittelshöfer ${ }^{1}$ ) hat z. B. 1879 aus der Literatur mehr als 40 derartige Fälle von partiellem Riesenwuchs sammeln können, und schon 5 Jahre später hat Lewin ${ }^{2}$ ) die Zahl auf 60 gebracht. Aehnliche Fälle sind ferner von Lesser ${ }^{3}$ ), Wagner ${ }^{4}$ ), Goldscheider ${ }^{5}$ ), George Humphry ${ }^{6}$ ) und möglicherweise von noch mehreren veröffentlicht worden. Dagegen gehört der Riesenwuchs zweier gleichseitiger Extremitäten oder gar einer ganzen Körperhälfte zu den grösseren Seltenheiten. Trotz energischer Literaturstudien haben Trélat und Monod $^{7}$ ) nur 12 solche Fälle aufgefunden, wozu sie dann selbst einen hinzufügen, und $\mathrm{Fischer}{ }^{8}$ ) hat sogar, ungefähr 10 Jahre später, die Sammlung nur mit 4 Fällen ver-

I) Langenbeck's Archiv. Bd. XXIV. 1879. S. 57 .

2) Charité-Annalen. IX. 1884. S. 652.

3) Schmidt's Jahrb. Bd. 201. 1884. S. 220.

4) Schmidt's Jahrb. Bd. 215. 1887. S. 191.

5) Arch. für Anat. und Physiol. 1889. H. 1-2.

6) Medico-chirurgical Transactions. London 1891. p. 165.

7) Archives générales de médecine. 1869. I. p. 536 et 676 .

8) Deutsche Zeitschr. f. Chirurgie. XII. 1879. No.1-2. Ș. 1. 
mehren können, während Lewin ${ }^{1}$ ) schliesslich nur mit 20 Fällen aufwarten kann.

Es lässt sich nicht leugnen, dass unser Fall mit der letaten Gruppe verwandt ist; ebenso sicher ist es aber, dass es sich bei ihm insofern um etwas mehr handelt, als wir es nicht nur mit einer abnorm starken Entwickelung grösserer Theile einer einzelnen Körperhälfte zu thun haben, sondern mit der Vergrösserung von Theilen beider Hälften. Es handelt sich mit anderen Worten um einen gekreuzten Riesenwuchs. Dieses ist gewiss eine sehr grosse Seltenheit; denn trotz eingehender Literaturuntersuchungen habe ich nicht mehr als 2 deutlich beschriebene Fälle von gekreuztem Riesenwüchs finden können, nehmlich einen Fall, den wir Friedberg ${ }^{2}$ ) verdanken, und einen anderen, der von Lewin') mitgetheilt ist. Keiner dieser Fälle kann sich jedoch mit dem unserigen messen. Vollständigkeitshalber füge ich noch hinzu, dass Dr. $\mathrm{Hahn}^{3}$ ) aus Stuttgart 1835 in einer deutschen medicinischen Gesellschaft ein 3jähriges Mädchen "mit angebornen monströsen Händen und monströser linker Brust" vorgezeigt hat; nähere Angaben fehlen jedoch.

Der Seltenheit wegen werde ich in aller Kürze die beiden anderen Fälle wiedergeben. Friedberg's Fall trägt die Ueberschrift:

Angeborner und fortschreitender Riesenwuchs des rechten Beines; consecutive Verschiebung des Beckens und Skoliose der Wirbelsăule. Angeborne Elepbantiasis arabum des linken Armes; Lipome an dem Rücken; venöse Teleangieltasien der Haut, chronischer Pemphigus u.s. w. (mit Abbildung).

Es handelt sich um ein 10 jäbriges Mädchen, das zum ersten Male im October 1853 untersucht wurde. Mager, kränkliches Aussehen, rachitisches Kind mit Kindergesicht und kleinem Kopfe. Die Haut überall welk, trocken, gelbbraun, bis anf die rechte Unterextremität und die Ulnarseite der linken Hand. Die Eltern und 7 Geschwister gesund. Die Mutter wäbrend der Schwangerschaft gesund. Nach ihrer Aussage waren schon bei der Geburt das rechte Bein und die linke Hand abnorm entwickelt; am Thorax zeigten sich venöse Teleangiektasien. Bei der Untersuchung bot das Kind die in der Uebersebrift angeführten Eigenthümlichkeiten dar, namentlich war die Vergrösserung des rechten Beines ausserordentlich augenfällig, indem dasselbe

1) a. a. 0.

2) Dieses Archiv. Bd. 40. 1867. S. 353.

3) Schmidt's Jabrb. 1835. Bd.5. S. 138. 
beinahe eben so gross war, wie der ganze übrige Körper (vom Trochanter bis zur Ferse $7 \frac{1}{2}$ rheinische Zoli länger, als das linke); das Bein glich an und für sich dem eines wohlgenährten kräftigen Mannes; der Fuss war aber so gross, dass er sogar bei einem solcben Manne riesig bätte genannt werden müssen. Die Formen waren durch Anschwellen verwischt. Die Nägel woblgebildet, abgesehen von ihrem Umfang.

Bei einer 2 Jahre später vorgenommenen Untersuchung konnte man constatiren, dass der Wuchs des rechten Beines nach allen Richtungen grösseren Fortschritt gemacht hatte, als der des linken. Das Kind starb 5 Jahre später an Phthisis.

\section{Lewin's Fall:}

Hypertrophia totius corporis cruciata. 26jähriger Lederarbeiter. Gesunde Familie. Obgleich von schwächlicher Constitution, hatte ihm nichts von Bedeutung gefehlt; er hatte z. B. weder Rachitis, noch Erscheinungen von essentieller Kinderlähmung gehabt, bis er vor einem Jahre Syphilis bekam und deswegen in Lewin's Klinik aufgenommen wurde. Hier fand sich an der linken Seite Hypertrophie des Gesichts mit Hyperämie und Hyperidrosis. Die Hypertrophie betraf namentlich die Weichtbeile, in geringerem Grade die Knochen (Os frontis, Os zygomaticum, Os maxillare sup. und inf.). Die Hyperämie rübrte von einer stärkeren Entwickelung der Gefässe der. Haut und der angrenzenden Schleimhäute her. Die Haut selbst natürlich. Die linke Seite des Halses umfangreicher, als die rechte, und der Carotispuls hier etwas stärker. Geringe Skoliose, links-convex, am mittleren Theile der Dorsalsäule. Der linke Arm um $2 \frac{1}{2} \mathrm{~cm}$ länger, als der rechte, und zwar liefert den Hauptantheil an dieser Verlängerung der Vorderarm mit $2 \mathrm{~cm}$; die Circumferenz durchgehends um $1_{\frac{1}{2}} \mathrm{~cm}$ grösser. Umgekehrt ist das rechte Bein länger, als das linke (vom Trochanter bis zum Malleolus externus $1 \mathrm{~cm}$ Unterschied), und voller (20 $\mathrm{cm}$ oberbalb der Patella beinahe $6 \mathrm{~cm}$, unterbalb der Patella $\frac{1}{2} \mathrm{~cm}$ Unterschied). Am Truncus, besonders an der linken Seite, und an den Nates venöse Hyperämie, an beiden Unterschenkeln Varicositäten. An der linken Seite des Halses Naevi melanodes, und an der linken Seite des Unterleibes, so wie am untersten Theile des Rückens linsen- bis erbsengrosse Vitiligines. Die Sensibilität ist, namentlich für Temperatur, rechtsseitig herabgesetzt. Der Patient schwitzt leichter und stärker an der linken, als an der rechten Seite. - Es fehlt Auskunft über den Anfang der Hypertrophie; der Verfasser, welcher eine grosse Abhandlung an diesen Fall knüpft, setzt voraus, dass die Hypertrophie congenital gewesen ist.

Obgleich diese beiden Fälle zu derselben Kategorie gehören, wie der unserige, so scheint doch keiner von ihnen im Grossen und Ganzen einen so universellen und so gut ausgesprochenen Riesenwuchs darzubieten, als der unserige, und ich glaube daher mit Recht den unserigen-für einen der schönsten Typen von ange- 
bornem Riesenwuchs, der je beobachtet worden ist, erklären zu dürfen. Er zeigt nicht allein mit Rücksicht auf die Volumenvergrösserung der verschiedenen Theile, sondern auch in jeder anderen Beziehung die Eigenthümlichkeiten, welche wir laut der umfassenden Untersuchungen von Trélat und Monod, wie auch von Wittelshöfer in Fällen von Riesenwuchs zu finden erwarten müssen. Ich werde in aller Kürze diese Eigenthümlichkeiten durchgehen.

Der Riesenwuchs ist immer angeboren. Es ist oft schwierig, den stringenten Beweis dafür zu liefern, weil das Individuum in der Regel erst später im Leben zur Beobachtung gelangt, wenn es wegen einer zufälligen Krankheit zum Arzte geführt wird. Solche Individuen sind nehmlich gewöhnlich trotz ihrer Deformität kräftige Wesen, die an und für sich dadurch nicht afficirt werden. In unserem Fall ist - dank der Protocolle der Gebäranstalt - kein Zweifel in dieser Beziehung möglich. Unser Fall bestätigt auch die Behauptung, dass der Riesenwuchs stets als isolirtes Factum vorkommt; weder vorausgegangene, noch gleichzeitige Generationen pflegen daran zu leiden. Es lassen sich keine Art von hereditärer Disposition, noch andere Anhaltspunkte in den Antecedentien der Eltern zum Verständnisse der Entstehung der Missbildung nachweisen. Als Curiosum schiebe ich hier noch ein, dass die Mutter in dem oben erwähnten, von Hahn mitgetheilten Falle meinte, die Deformität des Kindes stünde damit in Verbindung, dass sie sich während ihrer Schwangerschaft an einem Riesenknaben versehen hätte, welcher auf einem Jahrmarkte vorgezeigt wurde.

Unser Fall liefert demnächst ein gutes Beispiel dafür, dass die Vergrösserung nicht nur ein einzelnes Gewebe ergreift, sondern dass sowohl Bindegewebe, als Muskel-, Knochen- und Gefässgewebe daran Theil nehmen. In der Regel, wie auch in unserem Falle, sind das Knochengewebe und, vielleicht vorzugsweise, das Gefässgewebe am stärksten entwickelt. Die Harmonie der Grössenverhältnisse der einzelnen Theile ist in der Regel beibehalten; gewöhnlich sind es aber die meist peripherischen Theile der Extremität, welche am stärksten vergrössert sind, was durchgängig auch bei unserer Patientin der Fall ist. Nur am linken Beine ist der Oberschenkel verhältnissmässig mehr vergrössert, als der 
Unterschenkel (S. 109). Die Gelenke sind normal, wie auch bei unserem Kinde; ist die Bewegung beschränkt, so sind nur mechanische Hindernisse (Fett) daran Schuld. Auch die Haut pflegt natürlich zu sein, wie in unserem Falle; bisweilen kann sie vielleicht etwas grössere Festigkeit haben. Finden sich dann und wann Verdickungen der Haut, so rühren dieselben wabrscheinlich immer von secundären Leiden her. Die augenfälligsten Aenderungen erleidet jedenfalls das Gefässgewebe. In fast allen Fällen - und der unserige ist ein schönes Beispiel dafür - spielen ausgebreitete Teleangiektasien oder varicöse Venen eine hervorragende Rolle im Bilde. Die grösseren Gefässe - Arterien und Venen - sollen in den vergrösserten Theilen dickwandiger und zahlreicher sein. Das Fettgewebe ist ebenfalls häufig stark vergrössert, wahrscheinlich wegen des stark entwickelten Gefässgewebes, der daraus folgenden verstärkten Circulation und des dadurch vergrösserten Stoffwechsels. Die Fetthyperplasie kann förmlich excessive Grade erreichen; lipome können sich ausserdem in ihrer Folge entwickeln. Bei unserer Patientin findet sich jedoch nur eine stärkere Fettentwickelung an den Füssen; möglicherweise ist ihre Rachitis an dem sonstigen Fettmangel Schuld. Die Entwickelung der Nägel hält gleichen Schritt mit den betreffenden Phalangen; sie verhalten sich übrigens - wie auch in unserem Falle - ganz normal. Unter den mehr ungewöhnlichen Symptomen wird stärkerer Haarwuchs an den vergrösserten Theilen erwähnt; bei unserer Patientin finden sich auch einige kleine Haar-Inseln hinter den Schultergelenken und am Nacken. Etwas häufiger wird stärkere Schweisssecretion angeführt; die Rachitis unserer Patientin ist jedoch wahrscheinlich an der reichlichen Schweissabsonderung an ihrem Kopfe Schuld. In ganz vereinzelten Fällen wird Skoliose erwähnt, die sich, wie man annimmt, secundär in Folge des Uebergewichts der einen stärker entwickelten Seite ausbildet (siehe Friedberg's Fall); die geringe Kyphose, welche in unserem Falle vorhanden ist, muss wohl mit der Rachitis in Verbindung gebracht werden.

Das Nervensystem, wie auch alle höheren Sinne, sollen, soweit man es hat constatiren können, normal fungiren, und dieses scheint auch bei unserer Patientin der Fall zu sein. In dem 
einzigen Falle, in dem eine Section gemacht ist, war das Centralnervensystem normal. Es wird auch von allen Seiten hervorgehoben, dass die Intelligenz vollständig bewahrt wird. Wie wir aber bereits gesehen haben, war unsere Patientin vermuthlich in ihrer geistigen Entwickelung etwas zurück, da sie trotz ihrer 3 Jahre stumm und unreinlich, wie auch sonderbar scheu und ernsthaft war.

Die Eingeweide sollen nie etwas Abnormes darbieten; so ist es auch in unserem Falle.

Welche Zakunft wartet nun unserer kleinen Patientin? Nach den vorliegenden Erfahrungen sollte sie ja dieselbe Lebensfähigkeit haben, wie alle normal entwickelten Kinder. Ihre vergrösserten Körpertheile werden wahrscheinlich fortfahren, sich nach einem ähnlichen Maassstabe, wie bisher; zu entwickeln; sie haben vielleicht sogar Chancen, schneller und stärker, als die normalen, zu wachsen (vergl. Friedberg's Fall). Die vergrösserten Glieder werden ihr wahrscheinlich keine andere Schwierigkeit bereiten, als diejenige, welche durch ihr grösseres Gewicht verursacht werden mag.

Können wir eine Ansicht über den Ursprung des betreffenden Leidens haben? Es sind verschiedene Theorien darüber aufgestellt, die nach meiner Ansicht eigentlich nichts erklären, und die ich deshalb wiederzugeben keine Veranlassung finde. Eine Folgerung scheint mir indessen berechtigt: dass das Leiden schon im Eötalleben entsteht, weil es sich bei der Geburt ganz fertig zeigt, und dass es in genauer Verbindung mit der Entwickelung des mittleren Keimblattes, des Mesoderms, steht, welches, wie bekannt, das Bindegewebe, das Muskelgewebe, das Knochengewebe und das Gefässgewebe bildet, d. h. eben diejenigen Gewebe, welche am Entstehen des Riesenwuchses Theil nehmen. Da die Anomalie bei unserer Patientin eine so bedeutende Ausdehnung hat, und besonders, weil sie an beiden Seiten auftritt, ist die Ansicht anscheinend berechtigt, dass die erste Anlage zum Leiden auf eine sehr frühe Periode der embryonalen Entwickelung zurückgeführt werden muss. 